\title{
Variedades clonais de café Conilon para o Estado do Espírito Santo(1)
}

\author{
Scheilla Marina Bragança(2), Carlos Henrique Siqueira de Carvalho(3), \\ Aymbiré Francisco Almeida da Fonseca ${ }^{(2)}$ e Romário Gava Ferrão(2)
}

\begin{abstract}
Resumo - O objetivo deste trabalho foi selecionar e multiplicar clones de café Conilon (Coffea canephora Pierre ex Froehner) para obtenção de variedades clonais mais produtivas e de melhor qualidade. Foram selecionadas 267 plantas matrizes cujos parâmetros de seleção foram: produtividade, incidência de ferrugem (Hemileia vastatrix Berk et Br.) e mancha manteigosa (Colletotrichum sp.), arquitetura e vigor das plantas, tamanho e época da maturação dos frutos. Os clones selecionados foram avaliados em quatro experimentos, na Fazenda Experimental de Marilândia, pertencente ao INCAPER, em Marilândia, ES. O ensaio foi instalado em Latossolo Vermelho-Amarelo, no espaçamento de $3,5 \mathrm{~m}$ entre linhas e 1,5 m entre covas. Utilizou-se o delineamento de blocos casualizados, com quatro repetições e seis plantas por parcela. Dos clones selecionados, numa primeira fase, foram lançadas as primeiras variedades clonais de café Conilon, para o Estado do Espírito Santo, denominadas EMCAPA 8111, EMCAPA 8121 e, EMCAPA 8131, de ciclo de maturação precoce, médio e tardio, respectivamente, e com produtividades média de quatro colheitas oscilando entre 58 e 60 sacas de $60 \mathrm{~kg}$, superando em até $33 \%$ a produtividade da testemunha.
\end{abstract}

Termos para indexação: Coffea canephora, clones, propagação vegetativa, produtividade, melhoramento vegetal.

\section{Clonal varieties of Conilon coffee for the Espírito Santo State, Brazil}

\begin{abstract}
The objective of this study was to select and to multiply clones of Conilon coffee (Coffea canephora Pierre ex Froehner) to obtain clonal varieties with improved yield and quality. Two hundred and sixty seven mother plants were selected based on coffee grain yield, rust (Hemilea vastatrix) resistance, brown blight (Colletotrichum sp.) resistance, plant architecture, plant vigor, fruit size and time to fruit maturity. The selected clones were evaluated in four field experiments conducted on a Dark Yellow Latosol (Oxisol) at Instituto Capixaba de Pesquisa, Assistência Técnica e Extensão Rural (INCAPER), in Marilândia, ES, Brazil. Coffee trees were planted in rows $3.5 \mathrm{~m}$ apart with $1.5 \mathrm{~m}$ between plants in the rows. A randomized block design with four replications and six plants per plot was used. The selected clones, EMCAPA 8111, EMCAPA 8121, and EMCAPA 8131, early, medium and late maturity, respectively, were released for the State of Espírito Santo, Brazil. The productivity varied from 58 to 60 bags of $60 \mathrm{~kg}$ and was $33 \%$ greater than the control variety.
\end{abstract}

Index terms: Coffea canephora, clones, plant propagation, productivity, plant breeding.

\section{Introdução}

A espécie Coffea canephora Pierre ex Froehner, possui ampla distribuição geográfica, ocorrendo em

\footnotetext{
(1) Aceito para publicação em 7 de agosto de 2000 .

(2) Instituto Capixaba de Pesquisa, Assistência Técnica e Extensão Rural (INCAPER), Caixa Postal 62, CEP 29900-970 Linhares, ES. E-mail: bragancasm@tdnet.com.br, aymbire@incaper.es.gov.br

(3) Embrapa-Centro Nacional de Pesquisa de Milho e Sorgo (CNPMS), Caixa Postal 151, CEP 35970-701 Sete Lagoas, MG. E-mail: henrique@cnpms.embrapa.br
}

uma faixa ocidental e central tropical e subtropical do continente africano, da República da Guiné e Libéria ao Sudão e Uganda, com elevada concentração de tipos na República do Zaire (Chevalier, 1947, citado por Fazuoli, 1986). Segundo este mesmo autor, $30 \%$ da oferta de café comercializado no mercado internacional é proveniente desta espécie.

A oferta mundial de cafés robustas é proveniente principalmente da Indonésia, Costa do Marfim, Brasil, Uganda, Vietnã, Java e Índia. Segundo dados do Anuário Estatístico do Café (1998), na safra 1997/1998, a produção mundial de robusta foi de 31,04 milhões de sacas beneficiadas. No Brasil, a quase-totalidade das 
lavouras de café, genericamente conhecido por Robusta, é da cultivar Conilon (C. canephora), e o Espírito Santo é o maior produtor nacional, destacando-se ainda os estados de Rondônia, Minas Gerais, Mato Grosso, Bahia e Rio de Janeiro. Atualmente, mais de $60 \%$ do café produzido no Espírito Santo se origina desta cultivar, e responde por cerca de $70 \%$ da produção brasileira. Apesar de sua importância sócio-econômica para o Estado, a produtividade do café Conilon é baixa, isto é, aquém do potencial desta cultivar.

O café Conilon ( $C$. canephora) é diplóide com $2 \mathrm{n}=22$ cromossomos, e auto-incompatível, multiplicando-se através de fecundação cruzada. Esta incompatibilidade é do tipo gametofítica, e é ligada aos alelos $\mathrm{S}_{1}, \mathrm{~S}_{2}$ e $\mathrm{S}_{3}$ (Conagin \& Mendes, 1961; Berthaud, 1980). Devido à alogamia da espécie, observa-se grande heterogeneidade entre plantas de uma mesma lavoura, pois, as sementes obtidas não reproduzem necessariamente as características da planta-matriz. Esta variabilidade, que dificulta os tratos culturais e reduz a produtividade e a qualidade do café Conilon pode ser diminuída com a utilização da propagação assexuada de plantas-matrizes selecionadas.

A propagação assexuada por meio da multiplicação por estaquia foi estudada em alguns países da África, e sua viabilidade foi comprovada. Vallaeys (1952), citado por Dublin (1964), testou este método com o C. canephora, cultivar Robusta, e Dublin (1964), com o Coffea excelsa, encontrando resultados positivos. Em Madagascar, utilizando-se da seleção individual de plantas matrizes em lavouras comerciais, foi possível, através da propagação assexuada, a obtenção de um significativo aumento da produtividade das lavouras formadas (Snoeck, 1968).

Paulino et al. (1984), no Brasil, confirmaram estes resultados em C. canephora, cultivar Conilon, salientando que a mesma é particularmente adequada a este tipo de propagação, pois apresenta a vantagem de ser multicaule, permitindo a obtenção de grande número de estacas por planta, que são de fácil enraizamento.

No Brasil, Paulino (1980), com o objetivo de obter linhagens de café Conilon (C. canephora) com estabilidade de caracteres, avaliou 120 progênies desta cultivar, selecionadas em diversas lavouras do norte do Espírito Santo. Após seis produções, selecionaram-se as 30 melhores progênies, com produtividades que variaram de 11,95 sacas beneficiadas/1.000 covas a 45,02 sacas beneficiadas/ 1.000 covas; as progênies 63 e 160 foram consideradas as mais produtivas. Neste trabalho, as progênies foram classificadas quanto a época de maturação dos frutos, encontrando-se $49 \%, 29 \%$ e $22 \%$ de plantas de maturação precoce, média e tardia, respectivamente. Entretanto estas plantas não foram agrupadas por ciclo de maturação dos frutos.

O objetivo deste trabalho foi selecionar e multiplicar plantas matrizes de café Conilon (C. canephora) para a obtenção de variedades clonais que possibilitem a melhoria da produtividade e qualidade do café produzido.

\section{Material e Métodos}

Selecionaram-se 267 plantas matrizes em várias propriedades localizadas nos principais municípios produtores de café Conilon, no norte do Estado do Espírito Santo.

Para a seleção, adotaram-se os seguintes parâmetros das plantas matrizes: produtividade, incidência de ferrugem (Hemilea vastatrix Berk et Br.) e mancha-manteigosa (Colletotrichum sp.), arquitetura e vigor das plantas, tamanho, e época de maturação dos frutos.

Das plantas selecionadas no campo, foram retiradas as estacas, originando os clones, os quais foram multiplicados e reavaliados no viveiro da Fazenda Experimental de Marilândia, do INCAPER, em Marilândia, ES.

Os clones selecionados foram avaliados em quatro ensaios de competição, denominados Marilândia 86, Marilândia 87/1, Marilândia 87/2 e, Marilândia 88, instalados na Fazenda Experimental de Marilândia, ES. Os ensaios foram instalados em Latossolo Vermelho-Amarelo, textura argilo-arenosa, com as seguintes características químicas, à profundidade de $20 \mathrm{~cm}$ : $\mathrm{pH} 4,2 ; \mathrm{Al}$, $1,4 \mathrm{me} / 100 \mathrm{~cm}^{3}$; Ca, $0,4 \mathrm{me} / 100 \mathrm{~cm}^{3} ; \mathrm{Mg}, 0,4 \mathrm{me} / 100 \mathrm{~cm}^{3}$; K, 64 ppm; P, 4 ppm e matéria orgânica, 1,7\%.

As adubações e os tratos culturais foram feitos de acordo com as necessidades da cultura, e no plantio as mudas receberam $10 \mathrm{~g} /$ cova de $\mathrm{Ne} \mathrm{K}_{2} \mathrm{O}, 40 \mathrm{~g} /$ cova de $\mathrm{P}_{2} \mathrm{O}_{5}$, $0,5 \mathrm{~g} /$ cova de B, 2 g/cova de Zn e $10 \mathrm{~L} /$ cova de esterco de boi, além da calagem na área total e correção na cova. Na fase de produção, as plantas receberam, em média, $120 \mathrm{~g} /$ cova de $\mathrm{N}$ e $\mathrm{K}_{2} \mathrm{O}$, anualmente, e $30 \mathrm{~g} /$ cova de $\mathrm{P}_{2} \mathrm{O}_{5}$, em anos alternados, além das pulverizações com $\mathrm{ZnSO}_{4}(0,6 \%)$ e ácido bórico $(0,3 \%)$.

As capinas foram feitas somente nas linhas, com roçagens nas entre linhas (ruas). Os experimentos não foram irrigados. 
De acordo com a Carta Agroclimática do Estado do Espírito Santo, o Município de Marilândia é caracterizado como tendo os meses de janeiro, novembro e dezembro úmidos; os meses de fevereiro, março, abril e outubro parcialmente úmidos, e maio, junho, julho, agosto e setembro, secos. A temperatura média das máximas é de $33,5^{\circ} \mathrm{C}$, e a temperatura média das mínimas, de $13,9^{\circ} \mathrm{C}$. O município está situado à uma altitude de $150 \mathrm{~m}$, latitude de $19^{\circ} 24^{\prime}$ e longitude de 40³1'34" (Feitosa, 1986).

Neste trabalho serão enfocados os resultados do experimento Marilândia/86, no qual foram testados 77 clones, tendo-se como testemunha seis variedades de sementes. $\mathrm{O}$ delineamento experimental foi de blocos casualizados, com quatro repetições. A parcela experimental foi constituída por seis plantas, plantadas no espaçamento de $3,5 \mathrm{~m}$ entre linhas e $1,5 \mathrm{~m}$ entre covas.

Os clones foram avaliados durante seis anos (quatro produções), levando-se em consideração os seguintes parâmetros: produção, rendimento industrial (relação peso café maduro e café beneficiado), época de maturação dos frutos, altura e diâmetro da copa, peneira média, peneira maior que 13, porcentagem de grãos chatos, moca e concha.

Após quatro produções, os clones selecionados foram agrupados, por ciclo de maturação dos frutos, em precoce, médio e tardio, para a formação das variedades. A compatibilidade entre os clones foi determinada efetuando-se o cruzamento artificial entre os clones de cada variedade.

A análise de variância anual e conjunta foi feita em cada variedade clonal, e as médias foram comparadas pelo teste de Duncan, a $1 \%$ de probabilidade.

\section{Resultados e Discussão}

A análise de variância conjunta apresentou diferença significativa $(\mathrm{P}<0,01)$ em relação a tratamento, ano, e interação tratamento $\mathrm{x}$ ano nos grupos de clones de maturação precoce, média e tardia (Tabela 1).

Tabela 1. Resumo das análises de variância conjunta e anual dos dados médios de produção dos clones de café Conilon de ciclo de maturação precoce, médio e tardio, que originaram as variedades clonais EMCAPA 8111, EMCAPA 8121 e EMCAPA 8131, lançadas para cultivo no Estado do Espírito Santo.

\begin{tabular}{|c|c|c|c|c|c|c|c|}
\hline \multirow{3}{*}{$\begin{array}{l}\text { Fonte de } \\
\text { variação }\end{array}$} & \multicolumn{2}{|c|}{ Conjunta } & \multirow{3}{*}{ GL } & \multicolumn{4}{|c|}{ Anual } \\
\hline & \multirow[t]{2}{*}{ GL } & \multirow[t]{2}{*}{ QM } & & \multicolumn{4}{|c|}{$\mathrm{QM}$} \\
\hline & & & & 1989 & 1990 & 1991 & 1992 \\
\hline \multicolumn{8}{|c|}{ Ciclo precoce } \\
\hline Trat. (T) & 14 & $1.396,72 * *$ & 14 & $129,71 * *$ & 258,95 & $1.728,23 * *$ & $1.034,52 * *$ \\
\hline Ano (A) & 3 & $42.090,96^{* *}$ & - & - & - & - & - \\
\hline $\mathrm{T} \times \mathrm{A}$ & 42 & $584,90 * *$ & - & - & - & - & - \\
\hline Bloco & - & - & 3 & 52,38 & 174,32 & 469,69 & 233,10 \\
\hline Erro & 168 & 147,83 & 42 & 23,98 & 98,76 & 171,97 & 296,62 \\
\hline Média & & 52,37 & & 19,73 & 23,69 & 75,17 & 72,64 \\
\hline $\mathrm{CV}(\%)$ & & 23,22 & & 24,82 & 41,95 & 17,45 & 23,71 \\
\hline \multicolumn{8}{|c|}{ Ciclo médio } \\
\hline Trat. (T) & 42 & $1.691,04 * *$ & 42 & $155,47 * *$ & $450,00^{* *}$ & $1.031,94 * *$ & $1.770,00^{* *}$ \\
\hline Ano (A) & 3 & $111.481,05^{* * *}$ & - & - & - & - & - \\
\hline $\mathrm{T} \times \mathrm{A}$ & 123 & $572,13 * *$ & - & - & - & - & - \\
\hline Bloco & - & - & 3 & 50,57 & 41,51 & 393,46 & 33,96 \\
\hline Erro & 504 & 132,30 & 126 & 20,89 & 117,39 & 187,90 & 202,99 \\
\hline Média & & 50,17 & & 17,98 & 42,46 & 75,25 & 64,99 \\
\hline $\mathrm{CV}(\%)$ & & 22,93 & & 25,42 & 25,52 & 18,22 & 21,92 \\
\hline \multicolumn{8}{|c|}{ Ciclo tardio } \\
\hline Trat. (T) & 21 & $2.622,52 * *$ & 21 & $133,60 * *$ & $458,03^{* *}$ & $2.196,64 * *$ & $2.249,46^{* *}$ \\
\hline Ano (A) & 3 & $68.978,92^{* *}$ & - & - & - & - & - \\
\hline $\mathrm{T} \times \mathrm{A}$ & 63 & $805,07 * *$ & - & - & - & - & - \\
\hline Bloco & - & - & 3 & 8,92 & 283,78 & 650,38 & 613,23 \\
\hline Erro & 252 & 138,04 & 63 & 29,63 & 100,29 & 186,42 & 235,82 \\
\hline Média & & 53,45 & & 18,44 & 43,27 & 76,02 & 76,09 \\
\hline $\mathrm{CV}(\%)$ & & 21,98 & & 29,52 & 43,15 & 17,96 & 20,18 \\
\hline
\end{tabular}

**Significativo a $1 \%$ de probabilidade. 
Observa-se, nas Tabelas 2, 3 e 4, que as produtividades médias do 1을 2으, 3으 e 4 o ano foram de 19,73, $41,95,75,17$ e 72,64 sacas beneficiadas/ha com relação aos clones de ciclo de maturação precoce; 17,98 , $42,46,75,25$ e 64,99 sacas beneficiadas/ha, com relação aos clones de ciclo de maturação médio, e 18,44, $43,27,76,02$ e 76,09 sacas beneficiadas/ha, com relação aos clones de ciclo de maturação tardio, respectivamente. Esta interação significativa dos clones $\mathrm{x}$ anos mostra que a produção foi crescente até a quarta colheita.

A análise de variância anual apresentou diferença altamente significativa $(\mathrm{P}<0,01)$ entre os tratamentos estudados, e evidenciou uma variabilidade acentuada, na produção, entre os clones de ciclo de maturação precoce, médio e tardio (Tabela 1). Observou-se que a produtividade média de quatro colheitas variou de 21,51 sacas beneficiadas/ha a 71,99 sacas beneficiadas/ha, e 40 clones apresentaram produtividades abaixo daquela apresentada pela testemunha (Tabelas 2, 3 e 4), o que evidencia a importância do trabalho de seleção no programa de melhoramento do café Conilon.

Estes resultados confirmam os encontrados por Paulino (1980), mostrando, assim, a acentuada varia-

Tabela 2. Produtividade média (sacas beneficiadas/ha) dos clones de café Conilon que originaram a variedade clonal EMCAPA 8111, lançada para o Estado do Espírito Santo.

\begin{tabular}{llllll}
\hline Clone & 1989 & 1990 & 1991 & 1992 & Média $^{(1)}$ \\
\hline 02 & 32,25 & 42,94 & 103,62 & 78,00 & $64,20 \mathrm{a}$ \\
03 & 22,16 & 46,64 & 119,70 & 61,64 & $62,54 \mathrm{a}$ \\
112 & 29,79 & 48,52 & 84,00 & 84,64 & $61,74 \mathrm{a}$ \\
129 & 21,04 & 51,12 & 76,36 & 93,63 & $60,54 \mathrm{a}$ \\
26 & 17,57 & 41,50 & 92,74 & 81,84 & $58,41 \mathrm{a}$ \\
154 & 14,09 & 58,80 & 74,12 & 75,79 & $55,70 \mathrm{abc}$ \\
$104 \mathrm{a}$ & 20,60 & 39,33 & 71,51 & 84,94 & $54,09 \mathrm{abcd}$ \\
$104 \mathrm{~b}$ & 18,55 & 36,34 & 67,14 & 91,68 & $53,43 \mathrm{abcd}$ \\
29 & 23,75 & 46,43 & 65,73 & 77,45 & $53,34 \mathrm{abcd}$ \\
36 & 17,14 & 35,55 & 54,67 & 90,15 & $49,38 \mathrm{bcd}$ \\
SEM & 11,13 & 40,56 & 81,43 & 60,62 & $48,44 \mathrm{bcd}$ \\
17 & 19,25 & 40,55 & 72,63 & 54,12 & $46,64 \mathrm{~cd}$ \\
05 & 17,36 & 43,53 & 60,14 & 59,93 & $45,24 \mathrm{~cd}$ \\
47 & 18,23 & 31,48 & 73,31 & 52,22 & $43,81 \mathrm{~d}$ \\
118 & 13,03 & 25,94 & 30,39 & 42,94 & $28,08 \mathrm{e}$ \\
\hline Média & $19,73 \mathrm{D}$ & $41,95 \mathrm{C}$ & $75,17 \mathrm{~A}$ & $72,64 \mathrm{~B}$ & 52,37 \\
CV (\%) & 24,82 & 23,69 & 17,45 & 23,71 & 12,64 \\
\hline
\end{tabular}

(1)Médias seguidas da mesma letra, minúscula na coluna e maiúscula na linha, não diferem pelo teste de Duncan, a 5\% de probabilidade. ${ }^{(2)}$ Plantas provenientes de sementes. bilidade genética desta variedade, com reflexos na capacidade produtiva e nas características agronômicas.

Os clones que mais se destacaram na média de quatro colheitas foram: $02,03,112,129,26,154,104 a$, $104 \mathrm{~b}, 29$ e 36 , de ciclo de maturação precoce; 16,128 , $132,07,149,11,148,116,14,109 \mathrm{a}, 19,110 \mathrm{~b}, 120,110 \mathrm{a}$ e 201, de ciclo de maturação médio; e 99,143,46, 135,

Tabela 3. Produtividade média (sacas beneficiadas/ha) dos clones que originaram a variedade clonal EMCAPA 8121, lançada para o Estado do Espírito Santo.

\begin{tabular}{|c|c|c|c|c|c|}
\hline Clone & 1989 & 1990 & 1991 & 1992 & Média $^{(1)}$ \\
\hline 16 & 23,67 & 53,67 & 98,49 & 111,59 & $71,86 a$ \\
\hline 128 & 22,37 & 52,49 & 104,22 & 98,00 & $69,27 \mathrm{ab}$ \\
\hline 132 & 36,01 & 67,74 & 87,86 & 77,63 & $67,31 \mathrm{abc}$ \\
\hline 7 & 22,72 & 47,24 & 102,69 & 96,46 & $67,28 \mathrm{abc}$ \\
\hline 113 & 19,80 & 59,89 & 80,90 & 104,00 & $66,14 \mathrm{abc}$ \\
\hline 149 & 25,26 & 53,05 & 93,83 & 89,98 & $65,53 \mathrm{abc}$ \\
\hline 11 & 27,10 & 53,84 & 93,16 & 75,28 & $62,35 \mathrm{abcd}$ \\
\hline 148 & 8,35 & 27,49 & 93,06 & 107,51 & 59,10 bcde \\
\hline 116 & 18,90 & 59,04 & 105,20 & 53,04 & $59,04 \mathrm{bcde}$ \\
\hline 14 & 18,91 & 50,27 & 76,43 & 82,83 & $57,11 \mathrm{cdef}$ \\
\hline $109 a$ & 16,68 & 57,99 & 73,99 & 71,08 & $54,94 \mathrm{defg}$ \\
\hline 19 & 20,71 & 55,85 & 76,69 & 63,99 & $54,31 \mathrm{defg}$ \\
\hline 133 & 15,84 & 39,84 & 76,16 & 81,48 & $53,33 \mathrm{defg}$ \\
\hline 123 & 32,74 & 41,93 & 78,52 & 59,71 & $53,22 \mathrm{defg}$ \\
\hline $110 \mathrm{~b}$ & 19,93 & 38,85 & 78,31 & 75,08 & $53,04 \mathrm{defg}$ \\
\hline 120 & 19,11 & 45,79 & 91,21 & 51,67 & 51,95 defgh \\
\hline $110 \mathrm{a}$ & 16,64 & 44,82 & 66,98 & 78,53 & 51,74efghi \\
\hline 201 & 19,36 & 49,17 & 80,00 & 58,01 & 51,64efghi \\
\hline 191 & 17,38 & 42,16 & 62,49 & 84,23 & 51,56 efghi \\
\hline 50 & 19,04 & 41,99 & 77,32 & 65,14 & 50,87efghij \\
\hline 18 & 27,66 & 40,74 & 85,21 & 47,18 & 50,20 efghij \\
\hline 114 & 19,72 & 41,81 & 74,71 & 63,82 & 50,02 efghij \\
\hline 30 & 15,98 & 41,11 & 84,82 & 55,13 & 49,26 efghij \\
\hline $109 \mathrm{~b}$ & 18,63 & 46,27 & 74,52 & 56,14 & 48,89efghij \\
\hline $\mathrm{SEM}^{(2)}$ & 11,13 & 40,56 & 81,43 & 60,62 & 48,44efghijk \\
\hline 127 & 15,24 & 45,19 & 70,59 & 60,06 & 47,77fghijk1 \\
\hline 4 & 19,31 & 42,94 & 62,79 & 65,66 & 47,68fghijkl \\
\hline 131 & 23,63 & 45,26 & 90,26 & 26,66 & 46,45fghijklm \\
\hline 42 & 10,54 & 36,26 & 85,07 & 52,99 & 46,21ghijklm \\
\hline 24 & 15,79 & 45,88 & 66,56 & 56,14 & 46,09ghijklm \\
\hline 35 & 16,20 & 41,13 & 63,28 & 61,34 & 45,49ghijklmn \\
\hline 107 & 19,87 & 44,76 & 61,92 & 51,88 & 44,61ghijklmn \\
\hline 40 & 12,94 & 27,25 & 55,11 & 82,17 & 44,37ghijklmn \\
\hline 13 & 17,13 & 27,44 & 82,83 & 40,56 & 41,99hijklmno \\
\hline 140 & 10,42 & 27,15 & 57,82 & 68,79 & 41,05ijklmno \\
\hline 43 & 8,32 & 31,28 & 60,34 & 63,67 & 40,90ijklmno \\
\hline 21 & 13,32 & 33,22 & 55,61 & 59,95 & 40,52jklmno \\
\hline 108 & 9,99 & 18,58 & 54,16 & 68,43 & 37,79klmno \\
\hline 105 & 14,88 & 31,87 & 68,42 & 34,48 & $37,411 \mathrm{mno}$ \\
\hline 44 & 16,24 & 35,53 & 70,07 & 24,69 & $36,63 \mathrm{mno}$ \\
\hline 25 & 19,98 & 44,92 & 45,79 & 30,68 & 35,34 no \\
\hline 103 & 10,50 & 26,99 & 53,18 & 39,82 & 32,62 op \\
\hline 111 & 5,08 & 26,50 & 33,77 & 38,39 & $25,93 \mathrm{p}$ \\
\hline Média & $17,98 \mathrm{D}$ & $42,46 \mathrm{C}$ & $75,25 \mathrm{~B}$ & $64,99 \mathrm{~A}$ & 50,17 \\
\hline $\mathrm{CV}(\%)$ & 25,42 & 25,52 & 18,22 & 21,92 & 12,50 \\
\hline
\end{tabular}

(1)Médias seguidas da mesma letra, minúscula na coluna e maiúscula na linha, não diferem pelo teste de Duncan, a 5\% de probabilidade. (2)Plantas provenientes de sementes. 
100, 106, 144, $39139,32,45,49,153$ e 31 , de ciclo de maturação tardio. Esses clones geraram as variedades clonais EMCAPA 8111, EMCAPA 8121 e EMCAPA 8131, respectivamente (Tabela 5).

A variedade clonal EMCAPA 8111, constituída de dez clones, apresentou, na média de quatro colheitas, produtividade de 58 sacas beneficiadas/ha, com uma amplitude de variação de 49 a 64 sacas beneficiadas/ha; o rendimento industrial médio foi de 4,03, e o de peneira média, de 14 (Tabela 5). Os dez clones testados foram compatíveis entre si.

Tabela 4. Produtividade média (sacas beneficiadas/ha) dos clones de café Conilon que originaram a variedade clonal EMCAPA 8131, lançada para o cultivo no Estado do Espírito Santo.

\begin{tabular}{lrrrrl}
\hline Clone & 1989 & 1990 & 1991 & 1992 & Média $^{(1)}$ \\
\hline 99 & 18,36 & 59,44 & 101,74 & 108,44 & $71,99 \mathrm{a}$ \\
143 & 19,52 & 41,24 & 84,05 & 132,89 & $69,42 \mathrm{ab}$ \\
46 & 26,74 & 50,41 & 107,74 & 85,32 & $67,55 \mathrm{abc}$ \\
135 & 29,75 & 47,41 & 92,74 & 87,12 & $64,26 \mathrm{abc}$ \\
100 & 21,64 & 44,61 & 116,93 & 71,70 & $63,72 \mathrm{abcd}$ \\
106 & 20,46 & 64,21 & 98,82 & 65,45 & $62,23 \mathrm{abcd}$ \\
144 & 17,10 & 43,30 & 97,58 & 90,08 & $62,01 \mathrm{abcd}$ \\
39 & 20,57 & 38,53 & 83,13 & 99,41 & $60,41 \mathrm{abcde}$ \\
139 & 15,91 & 39,24 & 92,41 & 91,95 & $59,88 \mathrm{abcde}$ \\
32 & 22,01 & 54,07 & 75,41 & 83,44 & $58,73 \mathrm{abcdef}$ \\
45 & 25,37 & 60,31 & 72,25 & 76,13 & $58,52 \mathrm{bcdef}$ \\
49 & 12,17 & 46,46 & 66,86 & 101,46 & $56,74 \mathrm{bcdef}$ \\
153 & 15,92 & 44,28 & 96,94 & 65,85 & $55,75 \mathrm{cdefg}$ \\
31 & 22,73 & 43,88 & 66,21 & 70,07 & $50,72 \mathrm{defgh}$ \\
SEM & 11,13 & 40,56 & 81,43 & 60,62 & $48,44 \mathrm{efghi}$ \\
102 & 21,69 & 43,94 & 58,03 & 61,53 & $46,30 \mathrm{fghi}$ \\
23 & 14,18 & 40,04 & 47,53 & 72,97 & $43,68 \mathrm{hij}$ \\
142 & 23,30 & 41,03 & 38,68 & 62,35 & $41,34 \mathrm{hi}$ \\
12 & 5,27 & 26,21 & 60,20 & 69,60 & $40,32 \mathrm{hi}$ \\
134 & 16,71 & 33,83 & 46,48 & 50,28 & $36,83 \mathrm{i}$ \\
22 & 12,83 & 29,91 & 51,99 & 47,80 & $35,63 \mathrm{i}$ \\
158 & 12,29 & 18,95 & 35,37 & 19,43 & $21,51 \mathrm{j}$ \\
\hline Média & $18,44 \mathrm{C}$ & $43,27 \mathrm{~B}$ & $76,02 \mathrm{~A}$ & $76,09 \mathrm{~A}$ & 53,45 \\
CV (\%) & 29,52 & 23,15 & 17,95 & 20,18 & 14,94 \\
\hline
\end{tabular}

(1)Médias seguidas da mesma letra, minúscula na coluna e maiúscula na linha, não diferem pelo teste de Duncan, a $5 \%$ de probabilidade. ${ }^{(2)}$ Plantas provenientes de sementes.
A variedade clonal EMCAPA 8121, constituída de 15 clones, apresentou produtividade média de 60 sacas beneficiadas/ha, com uma amplitude de variação de 52 a 72 sacas beneficiadas/ha; o rendimento industrial médio foi de 3,96, e o de peneira média, de 15 (Tabela 5). Verificou-se, também, compatibilidade entre os 15 clones.

A variedade clonal EMCAPA 8131, constituída de 14 clones, apresentou produtividade média de 62 sacas beneficiadas/ha, com amplitude de variação de 51 a 72 sacas beneficiadas/ha; o rendimento industrial médio foi de 3,76, e o de peneira média, de 14 (Tabela 5). Os 14 clones foram compatíveis entre si.

As variedades clonais apresentaram alta produtividade, maior uniformidade de maturação, e frutos de maior tamanho. Na média das quatro produções EMCAPA 8111, EMCAPA 8121 e EMCAPA 8131 foram $29 \%, 33 \%$ e $33 \%$, respectivamente, mais produtivas que a testemunha, superando em até $857 \%$ a produtividade média do Estado do Espírito Santo (Tabela 5)

Estes resultados evidenciam a alta capacidade produtiva da cultivar Conilon, confirmando-se, assim, os resultados obtidos por Snoeck (1968) e Paulino et al. (1984).

Além disso, o método de propagação vegetativa utilizado neste trabalho, possibilitou a obtenção da primeira colheita comercial aos 24 meses de idade, superando em até $210 \%$ as variedades de sementes (testemunha). Este comportamento, também verificado em outras espécies, é inerente ao método de propagação assexuada, que determina uma diminuição no período de juvenilidade das plantas (Leopold \& Kriedemann, 1978). Isto ocorre porque a muda clonal de café Conilon é formada a partir de tecido diferenciado fisiologicamente, o que ocasiona a emissão dos ramos plagiotrópicos no início do seu de-

Tabela 5. Produtividade (sacas beneficiadas/ha) média das variedades clonais de café Conilon EMCAPA 8111, EMCAPA 8121 e EMCAPA 8131, em experimento não irrigado, lançadas para o cultivo no Estado do Espírito Santo.

\begin{tabular}{|c|c|c|c|c|c|c|c|c|c|c|c|}
\hline \multirow{2}{*}{$\begin{array}{l}\text { Variedade } \\
\text { clonal }\end{array}$} & \multirow{2}{*}{$\begin{array}{l}\text { Ciclo de } \\
\text { maturação }\end{array}$} & \multirow{2}{*}{$\begin{array}{l}\text { Época de } \\
\text { colheita }\end{array}$} & \multicolumn{5}{|c|}{ Produtividade $^{(1)}$} & \multirow[t]{2}{*}{ Amplitude ${ }^{(2)}$} & \multirow{2}{*}{$\begin{array}{l}\text { Índice } \\
\text { relativo }\end{array}$} & \multirow{2}{*}{$\begin{array}{l}\text { Peneira } \\
\text { média }^{(3)}\end{array}$} & \multirow{2}{*}{$\begin{array}{l}\text { Rendimento } \\
\text { industrial }\end{array}$} \\
\hline & & & 89 & 90 & 91 & 92 & Média & & & & \\
\hline E 8111 & Precoce & Até maio & 22 & 45 & 81 & 82 & 58 & $64-49$ & 129 & 14 & 4,03 \\
\hline E 8121 & Médio & Junho & 20 & 50 & 89 & 79 & 60 & $72-52$ & 133 & 15 & 3,6 \\
\hline E 8131 & Tardio & Julho/ago. & 21 & 48 & 90 & 82 & 60 & $72-51$ & 133 & 14 & 3,7 \\
\hline Testemunha $^{(4)}$ & Desuniforme & Maio/ago. & 10 & 38 & 77 & 57 & 45 & - & 100 & - & - \\
\hline
\end{tabular}

(1)Produtividade média obtida aos 24,36, 48 e 72 meses. (2)Limites superior e inferior de produtividade dos clones constituintes das variedades

${ }^{(3)}$ Clones selecionados com peneira média. ${ }^{(4)}$ Plantas provenientes de sementes de matrizes selecionadas. 
senvolvimento. Ao contrário, a muda formada a partir de sementes somente emitirá os ramos plagiotrópicos ou produtivos, na nona, décima e undécima axila foliar, determinando uma colheita comercial mais tardia.

Quando se comparou a produtividade entre as três variedades clonais, verificou-se que não houve diferença significativa. Entretanto, houve uma variabilidade acentuada na época de maturação dos frutos das variedades clonais avaliadas, com um diferencial de até três meses em relação à época de colheita. Esta característica das variedades clonais permitirá ao produtor programar a sua colheita, com uma melhor utilização da mão-de-obra na propriedade. Além disto, evitar-se-á a colheita antecipada do café ainda verde, prática às vezes adotada na tentativa de evitar o ataque da broca (Hypothenemus hampei), mas que contribui para diminuir o peso e a qualidade do café, com reflexos na rentabilidade da lavoura.

\section{Conclusões}

1. A seleção de clones por propagação assexuada permite aumentar a produtividade e melhorar a qualidade do café Conilon (Coffea canephora Pierre ex Froehner).

2. As variedades clonais EMCAPA 8111 , EMCAPA 8121 e EMCAPA 8131 são 29\%, 33\% e $33 \%$, respectivamente, mais produtivas que variedades formadas a partir de sementes.

3. As variedades clonais EMCAPA 8111 , EMCAPA 8121 e EMCAPA 8131 apresentam ciclo de maturação precoce, médio e tardio, respectivamente.

4. A obtenção de variedades clonais, com ciclo diferenciado de maturação dos frutos, melhora a qualidade do café.

\section{Referências}

ANUÁRIO ESTATÍSTICO DO CAFÉ. 4. ed. Rio de Janeiro : Coffee Business, 1998. 136 p.

BERTHAUD, J. L'incompatibilité chez Coffea canephora méthode de test et déterminisme génétique. Café, Cacao, Thé, Nogent-sur-Marne, v. 24, p. 167-174, 1980.

CONAGIN, C. H. T. M.; MENDES, A. J. T. Pesquisas citológicas e genéticas em três espécies de Coffea: autoincompatibilidade em Coffea canephora. Bragantia, Campinas, v. 20, n. 34, p. 787-804, 1961.

DUBLIN, P. Le bouturage du caféier Excelsa. Café, Cacao, Thé, Nogent-sur-Marne, v. 8, n. 1, p. 3-16, 1964.

FAZUOLI, L. C. Genética e melhoramento do cafeeiro. In: RENA, A. B.; MALAVOLTA, E.; ROCHA, M.; YAMADA, T. Cultura do cafeeiro: fatores que afetam a produtividade. Piracicaba : Associação Brasileira para Pesquisa da Potassa e do Fosfato, 1986. p. 87-113.

FEITOSA, L. R. Carta agroclimática do Estado do Espírito Santo. Vitória : EMCAPA, 1986. mapa color. Escala 1:400.000.

LEOPOLD, A. C.; KRIEDEMANN, P. E. Plant growth and development. New Delhi : McGraw-Hill, 1978. 545 p.

PAULINO, A. J. Comportamento de progênies da cultivar Conilon de Coffea canephora selecionadas no Espírito Santo. In: CONGRESSO BRASILEIRO DE PESQUISAS CAFEEIRAS, 8., 1980, Campos do Jordão. Anais... Rio de Janeiro : Instituto Brasileiro do Café-GERCA, 1980. p. $168-170$.

PAUlino, A. J.; PAUlini, A. E.; MATIELlO, J. B. Observações preliminares sobre a formação de lavouras de Coffea canephora $\mathrm{cv}$. Conilon através do enraizamento de estacas. In: CONGRESSO BRASILEIRO DE PESQUISAS CAFEEIRAS, 11., 1984, Londrina. Anais... Rio de Janeiro : Instituto Brasileiro do Café, 1984. p. 157-159.

SNOECK, J. La rénovation de la caféiculture malgache a partir de clones sélectionnés. Café, Cacao, Thé, Nogentsur-Marne, v. 12, n. 3, p. 223-235, 1968. 